\title{
Interfaces
}

\section{Le Tasse françois: Textes et images pour la réinterprétation de la Jérusalem Délivrée en France au temps de Marie de Médicis}

\section{Gabriele Quaranta}

\section{(2) OpenEdition}

1 Journals

\section{Édition électronique}

URL : http://journals.openedition.org/interfaces/284

DOI : 10.4000/interfaces.284

ISSN : 2647-6754

Éditeur :

Université de Bourgogne, Université de Paris, College of the Holy Cross

\section{Édition imprimée}

Date de publication : 1 janvier 2016

Pagination : 31-54

ISSN : 1164-6225

\section{Référence électronique}

Gabriele Quaranta, « Le Tasse françois : Textes et images pour la réinterprétation de la Jérusalem Délivrée en France au temps de Marie de Médicis», Interfaces [En ligne], 37 | 2016, mis en ligne le 19 mars 2018, consulté le 06 janvier 2021. URL : http://journals.openedition.org/interfaces/284 ; DOI : https://doi.org/10.4000/interfaces.284

\section{(c) (i)}

Les contenus de la revue Interfaces sont mis à disposition selon les termes de la Licence Creative Commons Attribution 4.0 International. 


\section{LE TASSE FRANÇOIS. \\ TEXTES ET IMAGES POUR LA RÉINTERPRÉTATION DE LA JÉRUSALEM DÉLIVRÉE EN FRANCE AU TEMPS DE MARIE DE MÉDICIS*}

\section{Gabriele Quaranta}

S'approcher de la langue et de la culture françaises ne fut ni simple, ni immédiat pour la nouvelle reine Marie de Médicis, qui était italienne et en plus florentine : elle avait grandi dans la ville qui avait imposé à une nation entière une langue raffinée, un moyen de communication partagé, une façon de se représenter l'univers (Dubost 117-132). Il fallut l'y pousser, comme Pierre Matthieu le raconte :

On la supplia de s'exercer dans la langue françoise, d'avoir aggréable qu'on luy parlast François, et de lire des livres François. Elle demanda qu'on lui en donnast. Le premier qui vint en ses mains fut Clorinde, ou l'Amante tuée par son amant qui lui fut d'autant plus aggréable qu'elle le cognoissoit par ce que le Tasse en a escrit (250).

Lire des textes en version originale pour en apprendre la langue était une pratique très répandue au $\mathrm{XVI}^{\mathrm{e}}$ et $\mathrm{XVII}^{\mathrm{e}}$ siècles, mais on pourrait aussi citer ce passage à propos des débuts du long mécénat artistique de la Reine, notamment au sujet de la réalisation autour de 1605-1606 du Cabinet de Clorinde à Fontainebleau (fig. 1), confiée au peintre Ambroise Dubois (Béguin, Dessins 235 ; Kerspern ; Wirth, Cabinet).

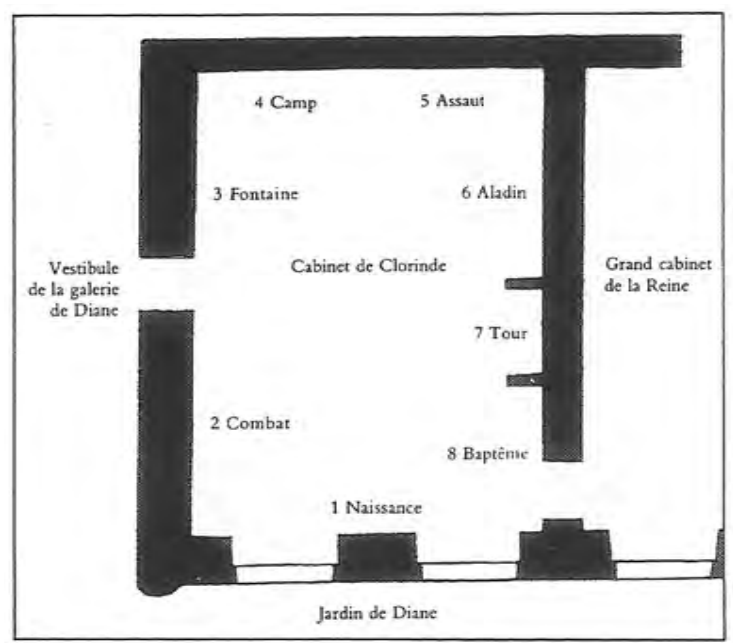

Figure 1 : Fontainebleau, Cabinet de Clorinde, Restitution de la disposition originaire des tableaux, par C. Samoyault-Verlet $(1987,1)$. 


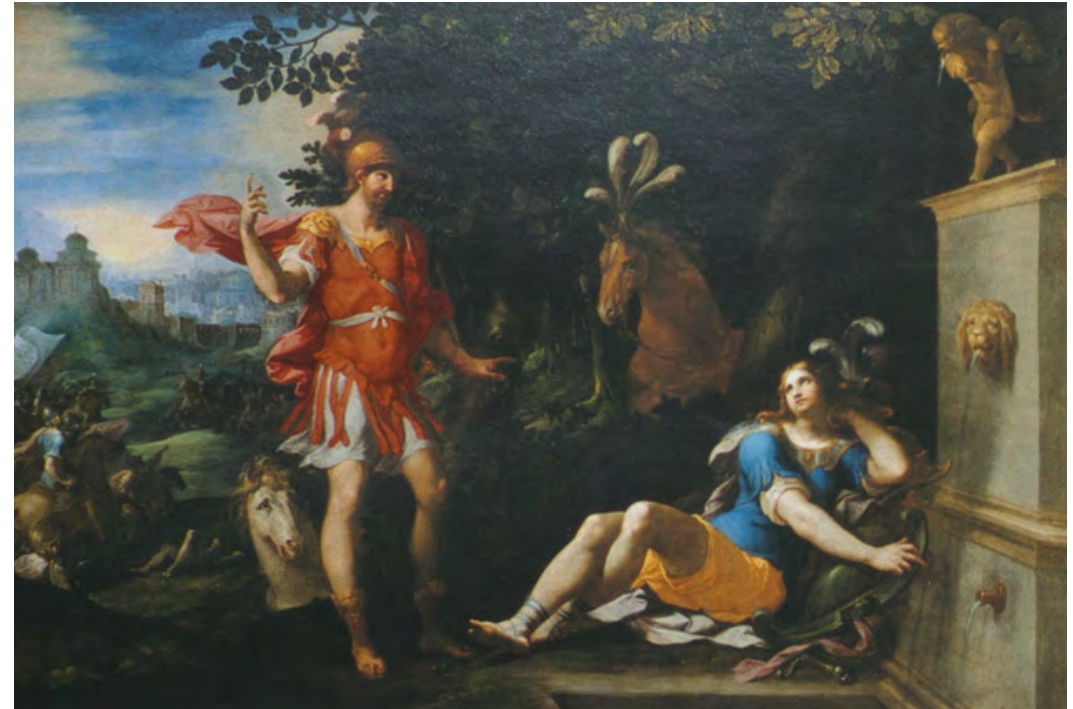

Figure 2 : Ambroise Dubois, Tancrède et Clorinde à la fontaine, 1604-1606, huile sur toile, Château de Fontainebleau, F.1980-3.
Le choix d'un sujet tassien pour un décor bellifontain

Le Cabinet de Clorinde était une pièce importante des logements royaux : on était obligé d'y passer pour entrer dans l'appartement de la Reine ou pour se rendre dans La Galerie de Diane voisine. Son décor a disparu au $\mathrm{XVIII}^{\mathrm{e}}$ siècle : ses riches boiseries entièrement perdues, il ne nous reste aujourd'hui que sept des huit ta-

bleaux du cycle principal, conservés ou connus par des copies, en tout cas déplacés de leur situation d'origine ${ }^{1}$. Une série de documents, et notamment l'Inventaire de Bailly (333-338), a pourtant permis à Colombe Samoyault-Verlet (Ambroise Dubois à Fontainebleau 2-3) de proposer une restitution convaincante de leur disposition (fig. 2).

* Ces pages constituent le résumé d'un chapitre de ma thèse doctorale, L'Arte del romanzo. Temi letterari nella pittura francese del Seicento (dal regno di Enrico IV alla reggenza di Anna d'Austria), soutenue en juin 2013 en régime de cotutelle internationale (Sapienza Università di Roma - Université Paris 1 Panthéon-Sorbonne). J'en profite pour remercier ici Mlle Marie Chaufour (Université de Bourgogne) d'avoir révisé mon texte, ainsi que Mlle Irene Zanot (Università Roma Tre) et Mme Stéphanie Prévost (Université Paris Diderot) pour leurs suggestions.

1 Cinq tableaux sont aujourd'hui à Fontainebleau : Combat de Tancrède et Clorinde, Tancrède et Clorinde à la fontaine, Tancrède devant les murs de Jérusalem, l'Assaut à Jérusalem, Clorinde devant Aladin, Baptême de Clorinde. La Naissance de Clorinde, qui ouvrait la série est perdue, et l'identification avec le dessin ENSBA, M 1375 proposée par Emmanuelle Brugerolles (260-262, cat. 84) reste, à notre avis, douteuse. Le tableau Clorinde incendie la tour nous est connu par deux copies, dont l'une est dans une collection particulière à Gambais, Château de Neuville et l'autre à Lier (Belgique), Musée Wuyts-Van Campen et Baron Caroly. Sur ce sujet voir en dernier Wirth, Cabinet 83-93. 
Le choix d'un sujet tassien de la part d'une reine d'origine italienne ne nous étonne guère, pourtant nous savons que ce décor bellifontain ne tirait pas son sujet du poème originel, mais d'une " adaptation » française, La Hierusalem Assiégée (fig. 3) : un roman d'Antoine de Nervèze dont nous ne connaissons aujourd'hui qu'une deuxième édition publiée à Paris en 1599, chez Anthoine du Brueil (Samoyault-Verlet, Ambroise Dubois à Fontainebleau 2).

Dans ce roman, qui ne retient du poème italien que l'histoire d'amour entre Tancrède et la belle amazone, Clorinde n'est pas la guerrière glaciale évoquée par le Tasse : elle partage dès le début les sentiments de Tancrède et leur amour refaçonne totalement l'histoire. La Rencontre à la fontaine (fig. 2), par exemple, qui dans le poème italien n'était que l'épiphanie foudroyante de la beauté guerrière de la Sarrasine, devient chez Nervèze un épisode d'amour courtois, une véritable joute amoureuse, fleurie de beaux mots, où les deux protagonistes, l'une songeant, l'autre mi-intimidé, mihardi, font connaissance et tombent amoureux l'un de l'autre.

Cette histoire d'amour se poursuit de part et d'autre des remparts de Jérusalem, à travers des échanges de longues lettres, des démonstrations de fidélité - un jour, Clorinde n'hésite pas à jeter en bas des murailles un soldat qui avait osé tirer sur Tancrède -, ou encore des rencontres fugaces au milieu des combats. Le récit tassien se transforme donc en roman typique de la fin de la Renaissance, tissé de dialogues fleuris et de politesses, de déclarations passionnées, de malentendus et de jalousies : finalement, la mort de Clorinde ne survient pas à cause de sa morgue chevaleresque mais suite à une erreur tragique aussi bien qu'illogique de Tancrède, qui ne parvient pas à reconnaître son amante au milieu des ténèbres.

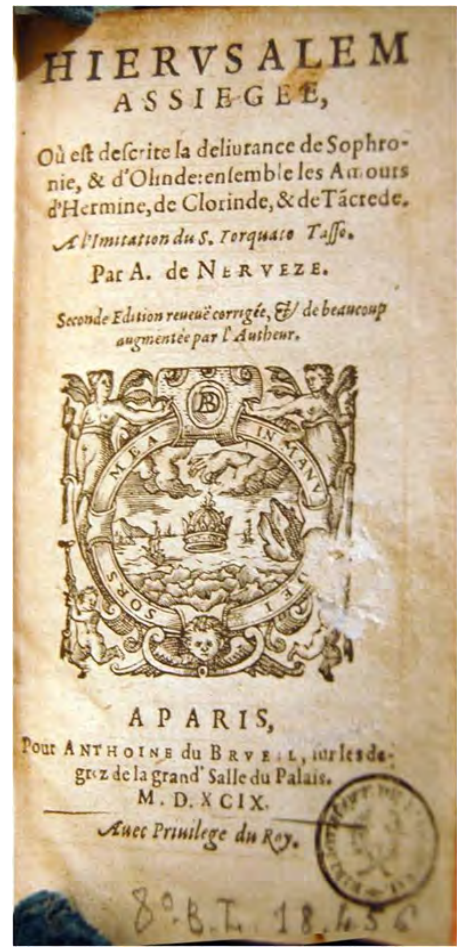

Figure 3 : Antoine de Nervèze, $L a$ Hierusalem Assiegée (...), Paris, chez Anthoine Du Brueil, 1599, page de frontispice (Paris, Arsenal, 8-BL18456).

Nous sommes ici très loin du monde du Tasse : le livre d'Antoine de Nervèze n'est pas du tout une «traduction » de la Jérusalem Délivrée; il s'agit plutôt d'une « imitation », comme son frontispice le déclare d'ailleurs, ou bien - dirait-on aujourd'hui - une dérivation, une reécriture. Nervèze choisit 
l'un des sujets principaux du poème et il en tire les épisodes éparpillés partout dans la narration, en les réunissant en forme de roman autonome. Mais en même temps, il les réécrit, il change leur signification et y ajoute des nouvelles aventures, en en supprimant d'autres. L'œuvre qui en ressort est donc d'origine tassienne mais tout à fait différente, tant pour ce qu'elle raconte, que par son esprit.

Il ne s'agissait pas d'un cas isolé, tout au contraire. Après une première édition de la Jérusalem Délivrée en langue originale à Lyon en 1581, et la publication de deux traductions intégrales - l'une en poésie par Jean de Vignau, l'autre en prose par Blaise de Vigenère, toutes deux sorties en 1595 -, nous connaissons une véritable série de réécritures à sujet tassien. En 1596, Pierre Joulet avait publié Les Amours d'Armide qui, de la même manière, s'était concentrée sur Renaud et Armide, l'autre couple protagoniste de la Jérusalem Délivrée. En 1597, le roman Clorinde ou l'amante tuée par son amant, que nous avons vu entre les mains de Marie de Médicis, fut publié. Il s'agissait d'un livre anonyme, mais considéré par certains comme la première, et inconnue, version du roman de Nervèze, car il y raconte un récit plus synthétique, mais en effet similaire (Simpson 80-85).

A côté de ces ouvrages, on peut compter aussi un grand nombre de réécritures pour le théâtre ou pour des sujets de ballet. Clorinde, tragédie d'Aemar de Veins fut encore publiée en 1599, toujours chez le même éditeur Anthoine du Brueil - un véritable spécialiste de ces ouvrages - et avec les mêmes illustrations que la Hierusalem Assiégée : la copie aujourd'hui conservée à la Bibliothèque de l'Arsenal porte sur son frontispice la signature d'Antoine de Nervèze, qui posséda le volume, et montre que ces auteurs se lisaient et s'imitaient les uns les autres. Nous apprenons grâce aux pièces liminaires que Aymard de Veins, sieur de Coudray, avait écrit deux autres pièces tassiennes, consacrées l'une à Olinde et Sophronie, l'autre - que l'on qualifie de tragicomédie - à Renaud et Armide. Elles ne furent apparemment jamais publiées, mais nous trouvons des ouvrages semblables tout au long des années 1600-1610 : par exemple, la tragédie anonyme La Sophronie publiée à Rouen (sans date, mais avant 1626) et à Troyes (en 1620), comportant elle aussi des illustrations.

Cette pratique d'adaptation ne concernait pas seulement la Jérusalem Délivrée, mais s'attachait à tout ouvrage considéré comme un chef-d'œuvre, donc un modèle et aussi un réservoir de personnages et de sujets romanesques : le Roland Furieux de Ludovico Ariosto compte en effet encore plus d'imitations, et cela dès les années 1570 (Cioranescu). Un tel discours est aussi valable pour le Pastor Fido de Guarini (Della Valle 24-36), les Ethiopiques d'Héliodore et bien d'autres encore. Parfois, on n'hésitait pas à mélanger de façon inattendue les différentes sources : la tragicomédie Clorinde, ou le sort des amants (1598) de Pierard Poullet est ainsi un « ouvrage monstre » qui mêle des personnages aux noms tirés du Tasse et de l'Arioste sur une intrigue proche de l'Aminta. 
Dans un contexte où une bonne partie de la noblesse - et surtout celle liée à la cour - n'avait aucune difficulté pour lire des textes en italien ou en espagnol, Françoise Graziani (206-209) a repéré les motivations d'un phénomène si répandu dans le besoin d'un passage d'un langage à l'autre plutôt que simplement d'une langue à l'autre : c'est-à-dire un passage de la langue poétique à celle de la prose. Habillé en roman aussi bien qu'en forme de pièce théâtrale, le récit épique originel devenait nettement plus proche du goût du public français, il en sortait francisé bien plus qu'à travers une simple traduction, comme cela est d'ailleurs montré par le sort des deux traductions intégrales de la Jérusalem délivrée : la version poétique de Jean de Vignau fut vite oubliée, alors que celle en prose de Blaise de Vigenère fut au contraire plusieurs fois réimprimée. Blaise de Vigenère, vieil homme de cour et traducteur expérimenté, connaissait bien le goût de ses lecteurs, et il en était également ainsi pour Jean Baudoin, qui allait choisir lui aussi la prose pour sa nouvelle traduction de 1626.

\section{Le Tasse relu par Nervèze à Fontainebleau}

Et justement, en revenant au récit tassien et à Fontainebleau, on pourrait se demander pourquoi Marie de Médicis opta pour le roman de Nervèze et non pas pour le poème du Tasse, si bien connu à la cour dans son originel italien et dans ses deux traductions intégrales.

Au tournant des années 1603-1605, Marie de Médicis commençait à jouer un rôle de plus en plus actif dans le contexte de la cour, qui l'avait jusqu'alors reléguée à la simple fonction de «mère des fils de France » (Dubost 228-248). Le Cabinet de Clorinde, on le disait au début, fut vraisemblablement sa première occasion de mécénat direct d'un ouvrage aux dimensions importantes : c'était également la première fois qu'on l'autorisait à proposer une image d'elle-même qui allait au-delà du simple portrait. Le récit ébauché par Nervèze lui permettait de dévoiler davantage de facettes de sa personnalité que l'héroïne du Tasse ne l'aurait permis : guerrière, certes, et sans doute femme forte, mais aussi amante fidèle, femme sûre de ses charmes et tout à fait à son aise, par sa grâce et par son esprit, sur la scène du grand théâtre du monde.

Alors que chez le Tasse, par exemple, Clorinde est une guerrière objet d'admiration mais tout à fait solitaire dans sa sombre intimité, Antoine de Nervèze soulignait son rôle de véritable chef des armées sarrasines, car le roi Aladin lui avait confié sans hésitation la défense de son royaume : cela permettait à Marie de pousser bien loin les allusions à ses prétentions, en se proposant même en bras droit du souverain, voire en véritable alternative, comme cela est montré par le choix de certains épisodes et le recours à des iconographies allusives. 


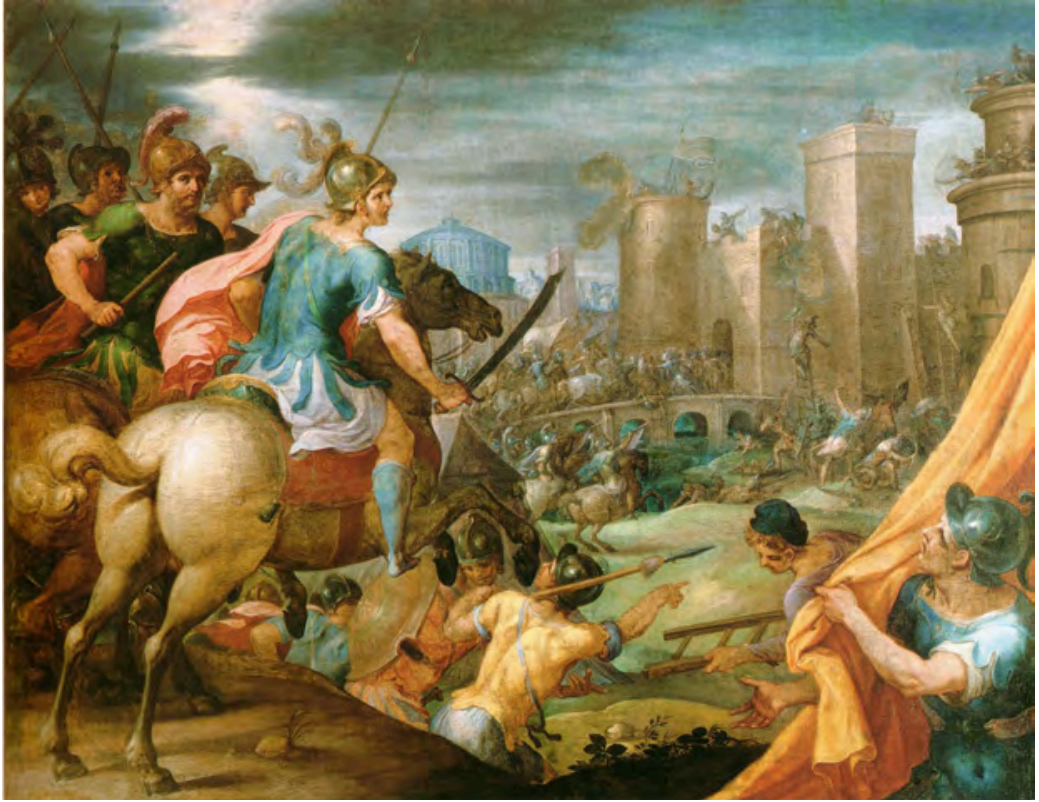

Figure 4 : Ambroise Dubois, Assaut à Jérusalem, 1604-1605, huile sur toile, Château de Fontainebleau, F.3232.C

leau, ils disparaissent presque dans un lointain second plan, alors que le premier est tenu par Godefroy de Bouillon qui se lance à la charge, cerné par ses officiers ${ }^{3}$. L'iconographie adoptée par Dubois est une

Après les quatre premiers tableaux, entièrement centrés sur Clorinde et sur son aventure sentimentale ${ }^{2}$, Ambroise Dubois s'arrête par exemple sur la représentation de l'Assaut à Jérusalem (fig. 4), un épisode où les deux protagonistes Tancrède et Clorinde sont repoussés au fond: Clorinde, armée de son arc, tire sur les chevaliers croisés qui cherchent à passer le pont d'entrée de Jérusalem ; tandis que Tancrède, au sommet de la tour d'assaut, mène ses soldats contre les remparts. Dans le ta-

2 Les premiers tableaux représentaient en effet en séquence la naissance de Clorinde (perdu), le premier combat avec Tancrède sous les remparts de Jérusalem, la rencontre à la fontaine, les échanges des lettres et de fidélités : ils étaient donc bien concentrés sur l'origine royale de la protagoniste et sur son histoire d'amour. Il nous appartient de noter que Nervèze oublie le détail pourtant crucial chez le Tasse de la naissance miraculeuse de la fille et de son abandon.

3 Le chevalier qui, le sabre à la main, charge à coté de Godefroy de Bouillon n'est pas Tancrède, comme on le dit normalement (Kerspern 145-146; Whirt 90-91) : le récit de Nervèze est très clair sur la position du Normand au sommet de la tour d'assaut. L'épisode raconte d'ailleurs que les deux amants combattent tout en restant attentifs à ne pas se blesser l'un l'autre. Godefroy n'est donc cerné que par ses officiers, et son geste risqué engendre l'étonnement du soldat au premier plan qui, sortant de sa tente, regarde, choqué, son commandant se lançant à la charge : un commentaire très clair du sens du tableau. 


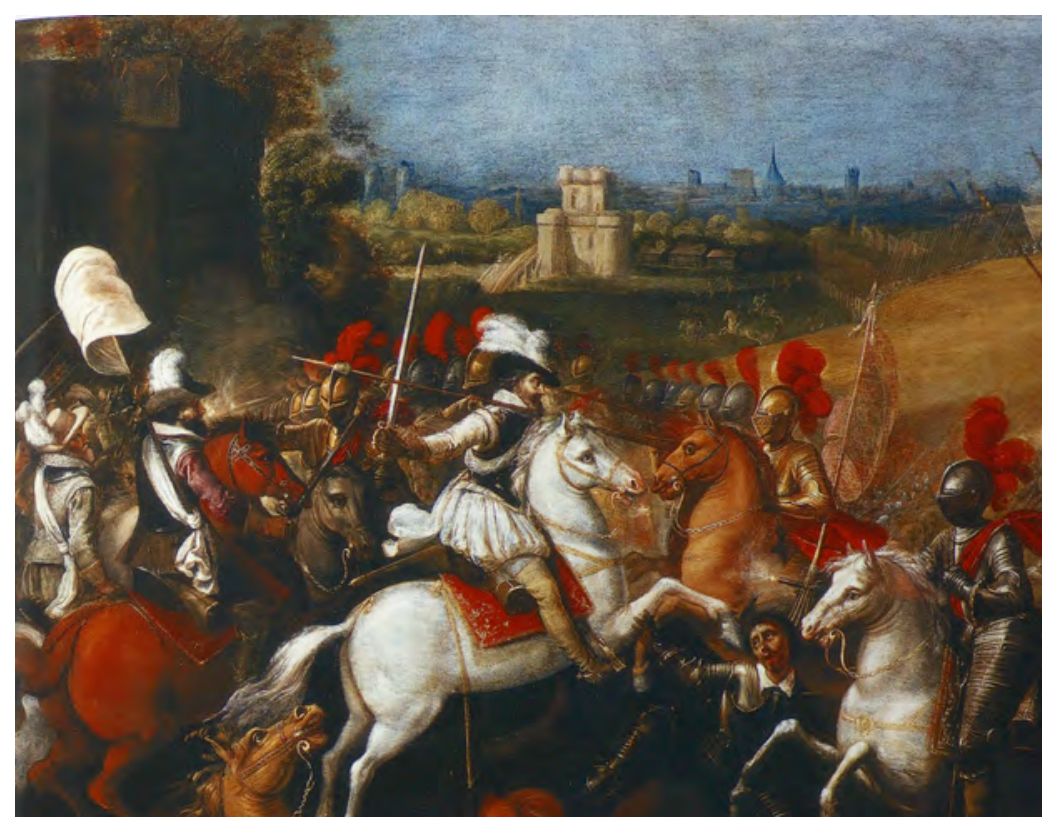

Figure 5 : Entourage de François Bunel, Henri IV à la bataille d'Arques, XVII siècle, huile sur bois, Pau, Musée National du Château, P.2007.6.1. allusion explicite aux batailles d'Henri IV (fig. 5), dont la représentation était installée juste à coté du Cabinet de Clorinde, dans la Galerie de Diane, mais aussi dans de nombreuses maisons de l'aristocratie française, qui y voyait la célébration de la hardiesse d'un roi se voulant tout d'abord chef de guerre et soldat parmi ses compagnons, n'hésitant pas à se mêler à la bataille (Wirth, Galerie ; Milovanovich). Or, c'est précisément par ce clin d'œil que le tableau de Dubois dévoilait son véritable message car, si le roman - comme le poème d'ailleurs - racontait l'échec de l'assaut par la faute de Clorinde, dont l'une des flèches blessait Godefroy et arrêtait sa charge, l'histoire de Nervèze n'obligeait pas à y consacrer un tableau spécifique et si concentré sur l'image du capitaine : la décision de le souligner se faisait donc porteuse d'une référence, peu cachée, à une suprématie du féminin sur le masculin, ou tout du moins à l'influence - ou au pouvoir - que Marie souhaitait exercer sur son époux. Le cycle de Clorinde d'ailleurs date d'un moment crucial de l'évolution politique de Marie de Médicis. Alors que la tradition de la cour de France, s'appuyant sur la loi salique, écartait la Reine de tout exercice du pouvoir - sauf dans le cas d'une régence (Solnon 163-211 ; Viennot, La France ; Cosandey) -, la maladie qui avait frappé Henri IV en 1603, avait fait craindre un décès à court terme : cette hypothèse, si elle devait se confirmer, signifiait une longue période de minorité du Dauphin, ainsi qu'une longue régence de Marie, qui, jusque-là, ne savait rien des affaires politiques. Cette situation amena ainsi le roi à souhaiter un rôle actif de son épouse au sein de la gestion de l'État : c'est à partir de cette année-là que Marie commença 


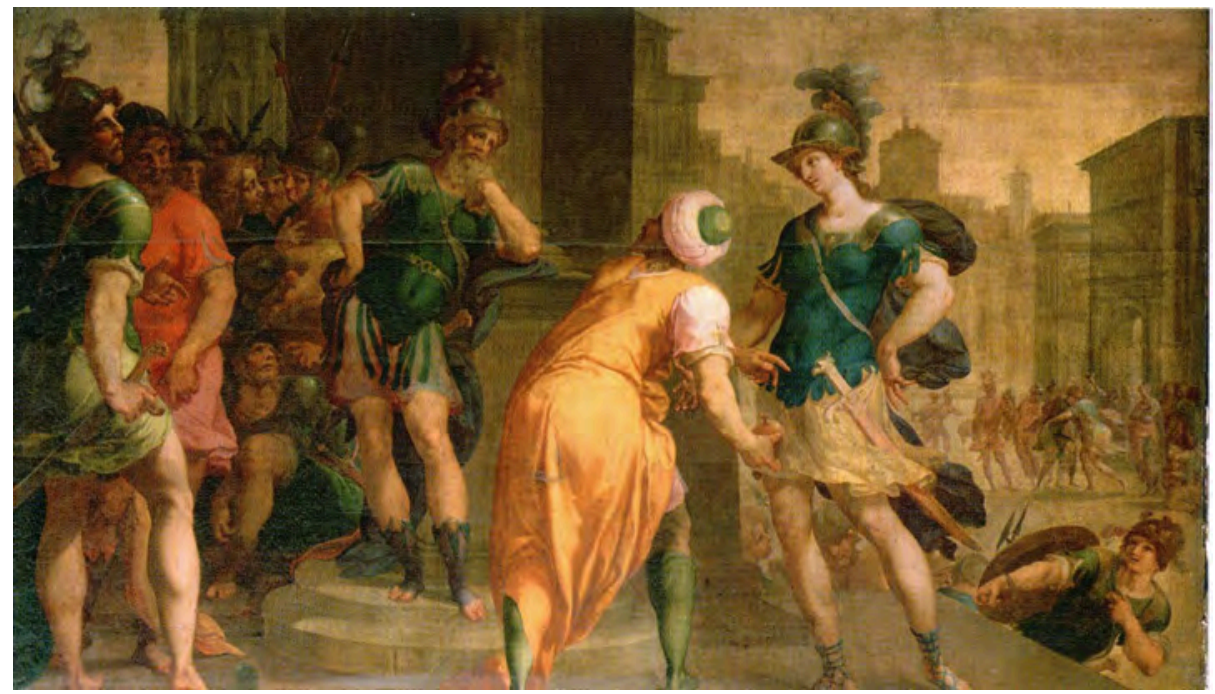

Figure 6 : Ambroise Dubois, Clorinde devant Aladin, 1604-1606, huile sur toile, Château de Fontainebleau, INV 4159.

à participer au Conseil et qu'elle fut tenue au courant des principales affaires de l'État (Dubost 235, \& 268-271) ; pourtant Henri IV continuait à lui refuser pour l'heure l'honneur du sacre et du couronnement (Bertière 60). Les années 1603-1604 virent aussi la disgrâce temporaire de la marquise de Verneuil, qui revint en 1606 en faveur auprès d'Henri IV, ce qui ne fut pas sans conséquences au sein des relations personnelles du couple royal. Le couronnement de Marie fut finalement différé jusqu'au mois de mai 1610, dix ans après leur mariage, alors que les reines précédentes avaient reçu leur sacre entre un et trois ans après leur union, comme c'était normal (Viennot, La France) ${ }^{4}$. Qui plus est, le couronnement de Marie fut concédé dans le contexte d'une reprise de la guerre contre les Habsbourg - donc d'un éloignement prolongé du monarque.

Pour en revenir à notre étude, la toile qui côtoyait l'Assaut à Jerusalem, Clorinde devant Aladin (fig. 6), montrant le conseil tenu dans Jérusalem suite à la bataille, était l'occasion de renforcer

$4 \quad$ Avec la seule exception de Louyse de Savoie, sacrée cinq ans après son mariage avec Louis XII : mais elle avait déjà reçu une première fois son sacre alors qu'elle était la femme de Charles VIII, et cela justement l'année suivant le mariage (Viennot, La France). 
le message du tableau précédent. Argan, dont le rôle est très important chez le Tasse, est ici, comme dans le roman de Nervèze, presque effacé, mélangé aux autres officiers, alors que Clorinde toute seule propose son plan d'incendier les machines de guerre ennemies. Au centre de la scène, installé sur des marches arrondies, Aladin, le roi au casque couronné, est assailli par le doute, ainsi que par la crainte de la perte de sa ville et par l'incertitude concernant le sort de la femme, qui est son seul rempart. L'opposition entre un souverain faible et hésitant d'une part, et une femme guerrière à l'esprit héroïque d'autre part se joue dans ce tableau : encore une fois, le contraste entre le masculin et le féminin s'éloigne du plan purement rhétorique pour devenir un argument qui pouvait s'appliquer au délicat équilibre sur l'échiquier politique de la cour, avec les rôles distincts du monarque et de son épouse.

Un tableau consacré à l'assaut nocturne de la tour, qui devait décorer la hotte de la cheminée mais que nous connaissons aujourd'hui seulement grâce à deux copies (Wirth, Cabinet 85), donnait une image iconique, voir emblématique de Clorinde : le sabre à la main, une grenade dans l'autre - selon les mots mêmes du roman de Nervèze -, celle-ci entraînait ses soldats à l'attaque du camp adverse, de manière irrépressible et ignivome. C'est là une célébration explicite de la femme guerrière qui n'est pas sans trancher avec la tradition ambigüe de l'amazone, figure fascinante et très présente dans la tradition littéraire et culturelle de la Renaissance française. Là, elle restait pourtant chargée d'une nuance négative, car, en principe, la femme ne devait pas se substituer à l'homme dans l'exercice de la guerre-l'ardeur militaire étant considérée aussi un indice explicite d'ardeur sexuelle(Steinberg 263-265). Si les années de guerres civiles avaient sans doute imposé aux femmes un rôle de premier plan, voire de véritables chefs de famille, cela ne concernait en tout cas pas le domaine militaire. Cela se cantonnait au cadre de l'organisation et de l'image familiales, du soutien logistique, et de la diplomatie (Viennot, Des Femmes d'État). C'est justement au début du XVII siècle que l'image de l'amazone commence à virer dans un sens franchement positif, qui lui sera typique ensuite, surtout pendant les années de la Fronde ; et c'était justement sous un tel masque qu'en 1601 le Ballet des Princesses des Isles, écrit par Jean Bertaut, célébrait la nouvelle Reine de France « que seul le plus grand roi de la terre a pu convaincre de renoncer à ses lois et à sa liberté » (Bertaut 652-654; Steinberg 269 ; Dubost 195). C'était évidemment jouer avec des codes connus, traditionnels et en même temps ambigus et délicats, où toute nuance devait être bien pesée. Le choix du roman de Nervèze s'explique ainsi pour sa relecture spécifique de l'image de Clorinde, qui joint à l'identité d'amazone tout à fait guerrière du personnage tassien, celle royale, galante et amoureuse d'une parfaite dame de cour. 
En glissant sur le moment de sa blessure mortelle, d'ailleurs très peu représenté en peinture, le cycle s'achevait juste à côté, entre la cheminée et l'une des portes d'entrée, sur le Baptême de la belle guerrière (fig. 7), tout en suivant encore une fois l'ample développement qu'Antoine de Nervèze faisait de l'épisode : Clorinde $\mathrm{y}$ prononçait un long monologue aux tons mystiques pendant que les cieux s'ouvraient devant ses yeux, même avant la bénédiction de Tancrède. Amante fidèle, meneuse de guerre hardie et héroïque, Clorinde endossait également le rôle de bonne chrétienne et de presque martyre.

Tandis que le roman de Nervèze se poursuivait avec bien d'autres épisodes, le cycle de Fontainebleau s'achevait sur ce tableau. Un tel final est très éloquent. En effet, toute l'attention devait se concentrer sur Clorinde, et ce faisant, le décor bellifontain opérait lui aussi la réédition d'un texte qui était déjà, en lui-même, une adaptation.

Le choix du roman de Nervèze s'explique alors bien sûr par le souci d'avoir une matière plus riche où puiser une image

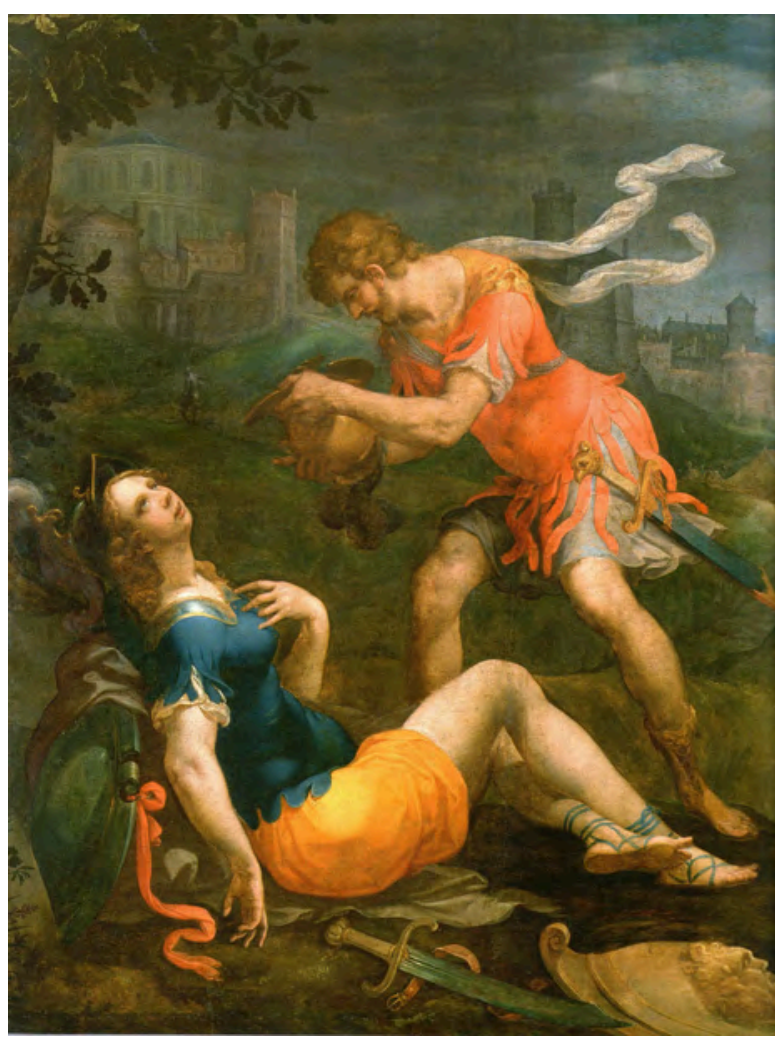

Figure 7 : Ambroise Dubois, Baptême de Clorinde, 1604-1606, huile sur toile, Château de Fontainebleau, F.1980-4.

plus complexe de la reine, mais il doit également être attribué - et il conviendra ici de se souvenir de la citation du père Matthieu d'où nous sommes partis - à un souci porté au « langage » : il s'agissait d'un choix franchement francophone de la part d'une souveraine qui, après ses premières années au nord des Alpes, montrait finalement la volonté d'abandonner ses origines italiennes et se proposait en véritable reine française pour un roi, une cour et un peuple français. L'histoire de Clorinde ne pouvait donc plus rester la saga poétique et mélancolique racontée par le Tasse : une simple « traduction » ne suffisait pas, elle devenait dorénavant un récit romanesque, en prose, un récit à la française. 
Textes et images pour la réinterprétation de la Jérusalem Délivrée en France au temps de Marie de Médicis

Ce « romanesque à la façon française », c'était aussi jouer avec les limites de la littérature : croiser les genres, mélanger les styles. C'était pousser sur l'amplification des épisodes et de leur pathétisme, forcer les récits en creusant en profondeur à la recherche de ce qui y était caché, de ce que les auteurs n'avaient pas voulu déclarer (Balsamo 296-302 ; Graziani 207-208). C'était bien ce que les réécritures promettaient, comme le dit de façon d'ailleurs explicite Pierre Joulet dans la dédicace à Mademoiselle de Lorraine des Amours d'Armide :

et d'autant qu'il me semble que ce Poète [le Tasse] a rendu quelques passions, sinon du tout muëttes, au moins un peu plus retenuës que leur naturel, ie leur ay laissé la voix libre, \& leur ay fait dire ce que i'ay pensé que l'on ressent au milieu du desplaisir, ou de la ioye qu'elles nous apporte(n)t (n.p.).

Si nous avons remarqué qu'à Fontainebleau, on n'avait pas retenu l'intégralité du roman de Nervèze, c'est justement parce que certains épisodes pouvaient sembler excessifs, peu convenables à la célébration d'une reine, voire tout à fait gênants.

On peut le constater grâce aux vignettes que l'on repère parmi les pages de ce roman illustré : de piètre qualité, mineures et presque enfantines, elles n'auraient pas retenu notre attention - et celle des contemporains -, si certaines d'entre elles n'apportaient pas des données intéressantes nécessaires à la compréhension du phénomène de l'appropriation française du récit tassien.

On est frappé, par exemple, par l'iconographie choisie pour la Lamentation de Tancrède sur le corps de Clorinde (fig. 8). La vignette, qui s'engage à représenter un épisode tout à fait négligé par les arts figuratifs ${ }^{5}$, suit de près le texte d'Antoine de Nervèze, où encore une fois un bref passage - quatre strophes et une poignée de vers en tout et pour tout chez le Tasse - se trouve amplifié sur

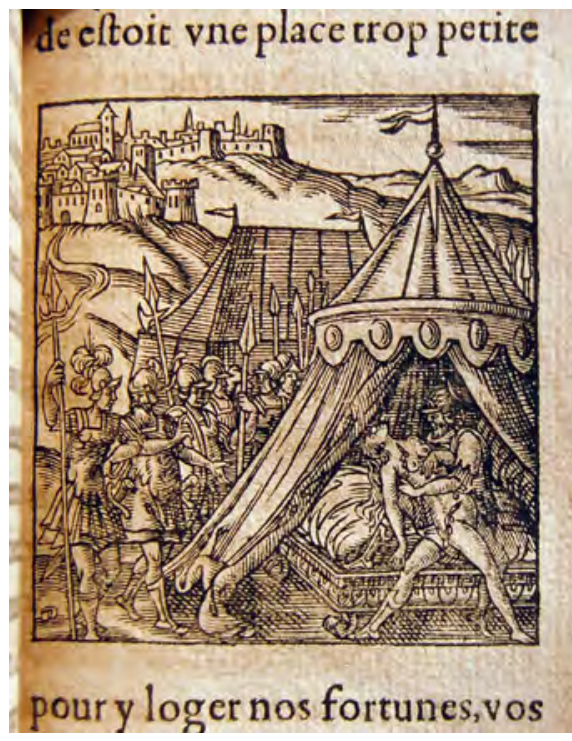

Figure 8 : Anonyme, Lamentation de Tancrède sur le corps de Clorinde, Figure dans Antoine de Nervèze, La Hierusalem Assiegée, Paris, chez Anthoine Du Brueil 1599, c. 127r (Paris, Arsenal, 8-BL-18456).

5 On peut citer ici le cas, lui aussi presque extraordinaire, du tableau du florentin Giovanni Bilivert, Tancrède contemple le corps de Clorinde (environ 1624, Florence, Palazzo Pitti) pour lequel je me permets de renvoyer ici à Fumagalli 73-84, 188-189, mais aussi à mon essai à paraître : Quaranta, Pentesilea e Clorinda. 
plusieurs pages. Dans son pavillon, sur son lit, Tancrède embrasse et caresse le corps désormais sans vie de Clorinde. Il pleure et plonge dans une lamentation longue et pathétique, que finalement l'écrivain commente ainsi :

A voir ces déplorables actio(n)s de Tancrede, on eust dict que sa doluleur, idolatroit ceste pauvre Amante, ou que luy mesme vouloit abuser de ce corps à la façon des Nigroma(n)ciens, tant il le cherit, \& le caresse. Mais ces caresses ont leur source, esloignée de la ioye, ce n'est qu'un fleuve de larmes, qu'un torrent de plaintes, \& qu'une rumeur de sanglots, \& de souspirs (131r-v)

De Nervèze, donc, joue avec un sujet très délicat et même gênant - la contemplation ou plutôt l'adoration d'un corps féminin dont la beauté reste séduisante même dans la mort -, là où le Tasse avait préféré s'arrêter à un plan très respectueux quoique allusif, et en cela le français est suivi par l'auteur de la vignette. Ce dernier représente Clorinde dans sa nudité, installée sur les genoux de Tancrède - le sang coule encore de sa blessure, mais son bras droit abandonné révèle qu'elle est morte -, et ainsi, n'hésite pas, tout en puisant dans la tradition picturale de la Renaissance, à adopter l'iconographie typique de l'allusion à l'acte sexuel. Il est frappant de confronter une telle image avec une représentation des amours d'Alexandre et Thalestris, reine des amazones, comme celle de Taddeo Zuccari au château de Bracciano (Iannuzzi 31).

En réalité, s'engager dans cette direction, pour l'auteur de la vignette, signifiait exhumer les origines mêmes de l'histoire de Tancrède et Clorinde. Le récit du Tasse n'était qu'une ultime récupération du mythe tragique d'Achille et Penthésilée et de leur amour qui éclot au moment même de leur combat meurtrier. Somme toute, il complétait une tradition de reprises bien établie dans la littérature italienne depuis la fin du Moyen-Age. Mais ses écrits montrent qu'il connaissait bien même les sources originales sur Penthésilée et les amazones (Dialoghi 454-497, 510-512), y compris la double tradition transmise par le récit latin du Excidium Troiae $(11,20)^{6}$ et par les commentaires du byzantin Eustache de Thessalonique $(169,208,8-13)$ qui racontaient la passion nécrophile du héros grec envers sa victime, violée après sa mort. Mais, alors que le Tasse avait seulement laissé paraitre en filigrane cette sombre tradition, dans l'entrelacement des mots allusifs qui brodaient le récit du combat nocturne (Chiappelli 56-65; Zatti 130-133), Nervèze, et plus encore son illustrateur anonyme, avaient permis à cette sombre histoire originelle de ressurgir, en parfaite résonnance avec le goût du macabre qui devait se répandre dans la littérature française pendant les deux décennies suivantes (Rousset ; Macchia 129-130).

6 Sur ce récit et sur ses nombreux manuscrits voir De Marco 36-56. 
Une deuxième vignette retient notre attention car elle nous permet d'évaluer la diffusion de la Hierusalem Assigée et sa fortune chez les artistes.

Après l'enterrement de Clorinde, Tancrède se rend sur son tombeau (fig. 9) : ici il est rejoint par Pierre l'Hermite. Le vieux moine joue chez Nervèze un rôle bien différent : alors que chez le Tasse (Gerusalemme Liberata XII, 85-88), il réprimande le chevalier encore convalescent dans son lit, en lui rappelant la Croisade et ses devoirs de soldat chrétien ; dans les pages du roman français, il le rencontre près du tombeau de Clorinde en lui apportant consolation (Nervèze 129r-131v).

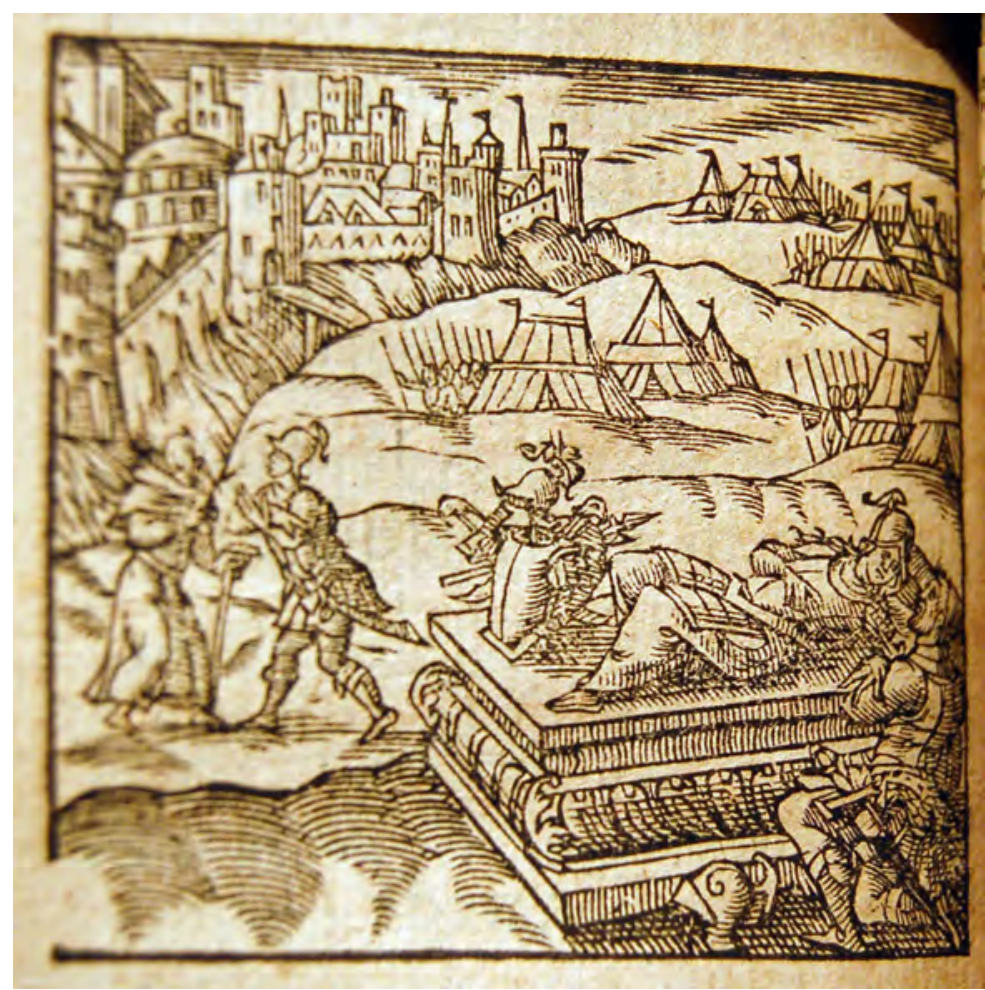

Figure 9 : Anonyme, Tancrède pleurant sur la tombe de Clorinde, Figure dans Antoine de Nervèze, La Hierusalem Assiegée, Paris, chez Anthoine Du Brueil, 1599, c. 132v (Paris, Arsenal, 8-BL-18456). 
C'est un changement très intéressant, évidemment enregistré par l'illustration. Elle nous permet ainsi d'établir une confrontation avec un dessin de Laurent de La Hyre (University of Michigan Museum of Art, 1970/2.89), qui représente une scène tout à fait similaire (fig. 10). Au début de sa carrière, vers 1624-1626, le jeune artiste s'était attaché à la traduction graphique de certains sujets romanesques comme Panthée sur lequel il allait revenir plus tard en peinture -, Alceste, et bien sûr la Jérusalem Délivrée à laquelle deux séries de dessins avaient été consacrées : l'Histoire de Renaud et Armide et l'Histoire de Tancrède et Clorinde (Rosenberg-Thuillier 115)

Mais le dessin aujourd'hui conservé au University of Michigan Art Museum montre bien que l'artiste faisait référence au roman de Nervèze, auquel il faudra rattacher aussi l'autre dessin possédé par le même musée (1970/2.88), qui ne montre pas - comme on le dit fréquemment - l'Aventure dans la forêt enchantée - épisode rejeté par Nervèze - mais plus vraisemblablement la Rencontre à la fontaine (fig. 11).

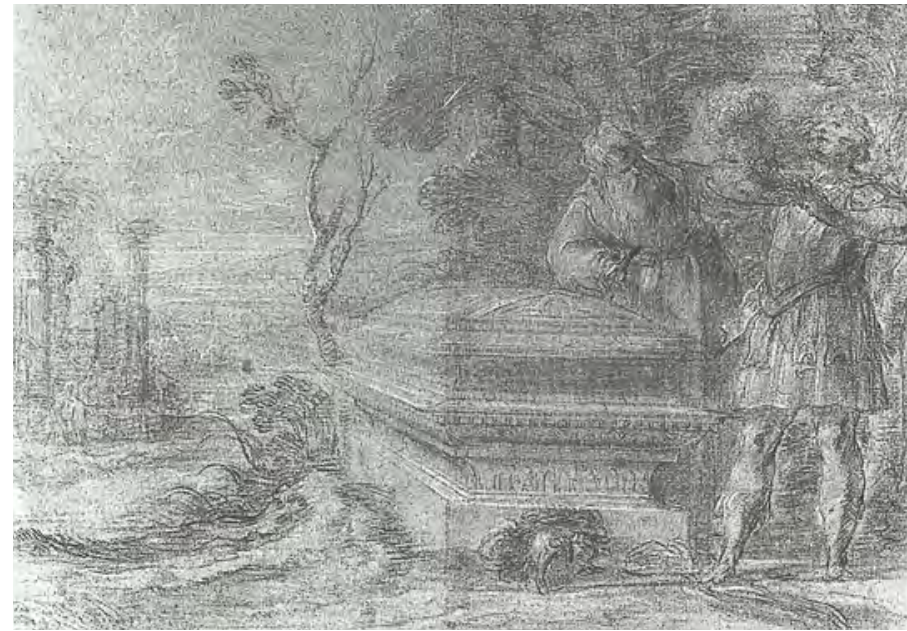

Figure 10 : Laurent de La Hyre, Tancrède pleurant sur la tombe de Clorinde, Ann Arbor, The University of Michigan Museum of Art, 1970/2-89.

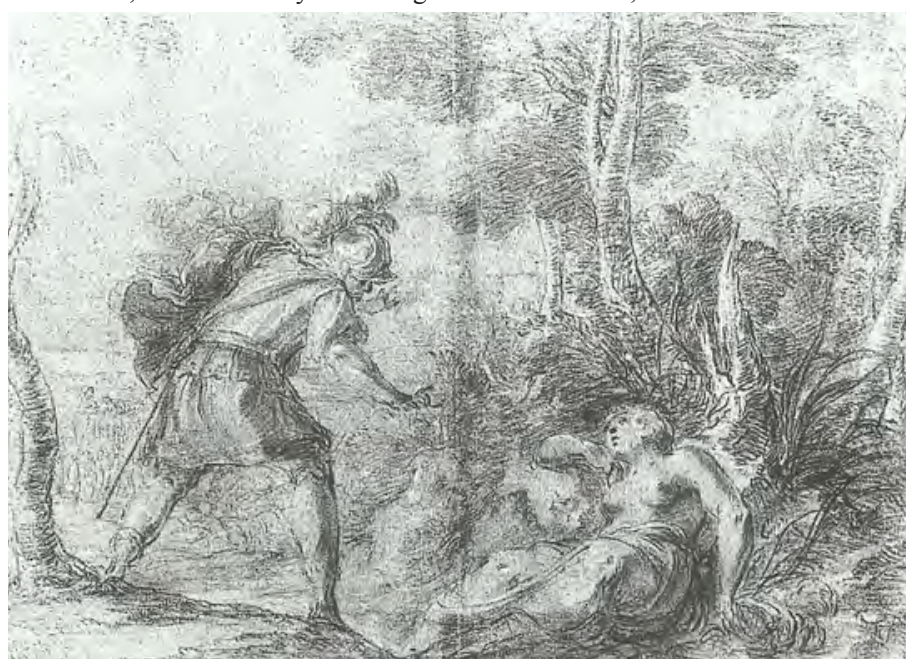

Figure 11 : Laurent de La Hyre, Tancrède dans la forêt enchantée (?), ici proposé comme Tancrède et Clorinde à la fontaine, Ann Arbor, The University of Michigan Museum of Art, 1970/2-88. 
Les deux séries tirées du récit tassien par La Hyre comptaient respectivement quatorze et douze dessins à l'origine. Elles ont été mélangées et dispersées suite à différentes ventes depuis le XVIII ${ }^{\mathrm{e}}$ siècle : pourtant, rapprocher l'une d'entre elles à une source si spécifique et pourtant si différente du poème italien nous permet de regrouper de façon nouvelle la poignée de dessins aujourd'hui connue, et cette démarche sera sans doute utile dans la recherche des autres (Quaranta, Arte del romanzo 73-82).

Avec Laurent de La Hyre nous sommes désormais vers la moitié des années 1620 : le roman d'Antoine de Nervèze avait été publié à plusieurs reprises jusque en 1623 et il influençait encore les arts figuratifs. Mais en effet, on sortait d'une décennie où les sujets tassiens avaient reçu les honneurs de la cour de France.

\section{Clorinde et la mythologie de la Couronne de France : perspectives rivales}

Le Cabinet de Clorinde joua sa part dans le choix de la couronne française de confier son autoreprésentation non seulement à la mythologie et à l'allégorie, mais aussi à des sujets littéraires. Après la tentative de renouer avec une filière «épique » nationale en récupérant La Franciade de Pierre de Ronsard dans la galerie du Château de Saint-Germain-en-Laye (Cordellier) - tentative restée isolée et infructueuse -, on se tournait désormais vers les romans. A la fin des années 1610, la Couronne faisait encore appel à Ambroise Dubois pour le nouveau décor de la Chambre Ovale de Fontainebleau (Sarant 25-37 ; Véron-Dénise 91-109) : dans cette salle, qui avait vu la naissance du Dauphin, la décoration picturale était tirée des Ethiopiques d'Héliodore et était chargée de la valeur dynastique ajoutée à ce roman grec par le traducteur Jacques Amyot (Quaranta, Arte del romanzo 8-11), qui, aux débuts des années 1570, en avait fait le modèle rhétorique central dans son Projet pour une éloquence royale (Plazenet 140). A côté du Tasse, Héliodore aussi allait jouir d'une fortune remarquable à la cour et chez la noblesse, fortune qui nous permet d'apercevoir le changement de sens idéologique attribué à ces différents récits.

D'ailleurs, ce n'est pas par hasard si, à la moitié des années 1610, après la mort d'Henri IV, après le succès du double mariage « espagnol » de Louis et d'Elisabeth, à l'apogée de sa Régence et désormais sûre de son pouvoir, Marie de Médicis n'hésita pas à reprendre la tradition originelle de la Jérusalem Délivrée, et non ses imitations, dans le décor de son nouveau cabinet de reine-régente au Palais du Louvre (fig. 12). Dans cette salle, destinée aux réunions du Conseil de la couronne, on confiait à Sophronie et à l'histoire de son martyre heureusement raté (Gerusalemme Liberata II, 14-53) l'image d'une femme belle, sage et fidèle, prête au dernier sacrifice pour l'amour de son peuple : l'image 
parfaite d'une souveraine qui assurait la conservation de ses sujets pendant la minorité du Dauphin. Ce décor, confié à une équipe de jeunes peintres qui suivaient de près la tradition de Dubois - qui venait de mourir au moment de l'ouverture du chantier - a entièrement disparu (Erlande-Brandeburg 105-113). Il ne nous est connu que d'après des descriptions antiques, notamment par Félibien et Sauval, et grâce à une série de dessins retrouvés à partir des années 1960, suite aux études pionnières de Sylvie Béguin (Béguin, Guillaume Dumée 234-243 ; Scaillerez 156-163). Réalisé alors que l'on publiait

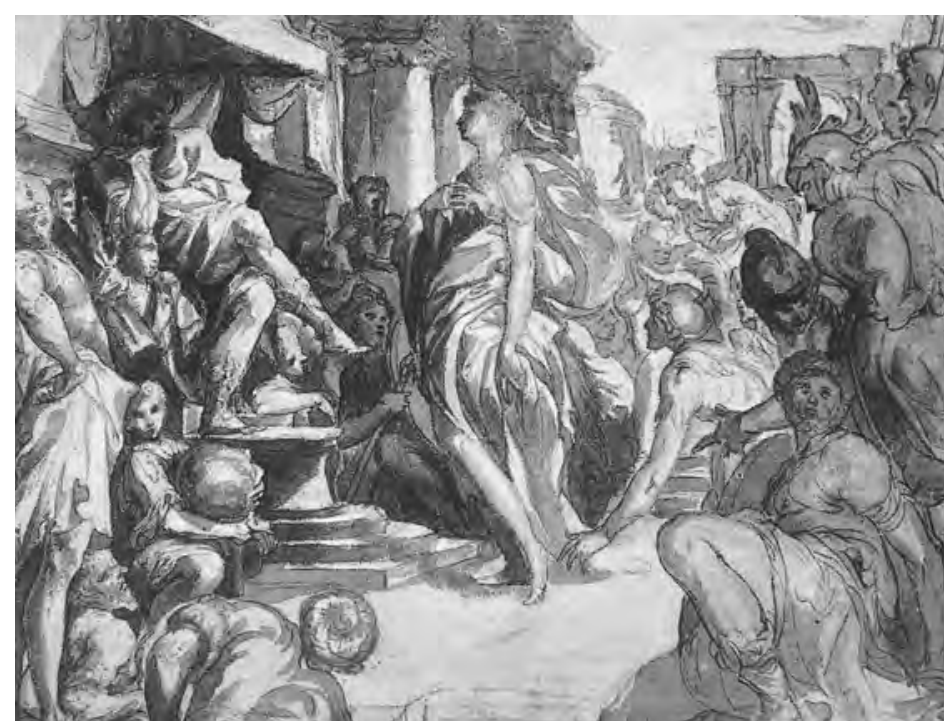

Figure 12 : Gabriel Honnet, Sophronie qui s'accuse devant Aladin, vers 16131615, dessin, Paris, ENSBA, M.904.

et lisait encore le roman de Nervèze, le cycle du Louvre revenait sur le récit du Tasse car, à ce momentlà, Marie n'avait plus rien à prouver à une cour qu'elle pensait dominer aisément, et la série romanesque était accompagnée par une deuxième à sujet dynastique, consacrée à la gloire de la maison de Médicis à travers la représentation des hauts faits des ancêtres (Bassani, Patch 92).

En même temps - nous sommes ici en 1614 -, les Jésuites proposaient au jeune Louis XIII des pièces théâtrales dont les textes demeurent inconnus mais dont les titres Godefroi de Bouillon et Clorinde nous disent l'importance donnée aux sujets tassiens dans le cadre de l'éducation du futur souverain (Quaranta, Arte del romanzo 66).

Ainsi, on ne s'étonne pas si le même Louis XIII voulut renverser totalement le sens de ces histoires lors de sa prise de pouvoir. Pendant les années 1617-1619, pendant la Guerre de la mère et $d u$ fils, les ballets de cour mis en scène à l'occasion du Carnaval (McGowan 101) relançaient l'image d'une nouvelle structure de pouvoir, en s'appuyant une fois encore sur des sujets littéraires, pour ainsi dire, revus et corrigés. 
Le 29 février 1617, trois mois avant l'assassinat de Concini, la Cour put assister au Ballet de la Délivrance de Renaud. Les textes étaient du poète Estienne Durand, ainsi que de René Bordier et Pierre Guédron ; la musique était de Jacques de Belleville et Gabriel Bataille; et les «machines » de Thomas Francini : l'ancienne équipe des ballets de Marie de Médicis. Louis XIII y jouait deux rôles, le Démon $d u \mathrm{Feu}$ et Godefroi de Bouillon, alors que son favori De Luynes était Renaud. L'histoire se déroulait comme le Tasse l'avait conçue, sauf qu'à la fin, il n'y avait pas de place pour une réconciliation entre le chevalier et la magicienne, qui se trouvait défaite et abandonnée, tandis que le premier, héroïque, se retrouvait avec ses compagnons autour du capitaine Godefroy. Le ballet représentait un pouvoir masculin qui échappait à une domination féminine, un système chevaleresque ordonné autour de son chef qui remplaçait un désordre de nature magique et démoniaque (Careri 204-216).

L'année suivante, en 1618, le ballet de La folie de Roland, tiré de Ludovico Ariosto, mis en scène par la même équipe, proposait l'histoire de l'esprit perdu du paladin et retrouvé grâce à l'intervention d'Astolphe, joué par De Luynes qui, après la disparition de Concini, trouvait ici la consécration de son nouveau rôle à la cour.

Et finalement, de façon encore plus claire, en février 1619 on renversait la rhétorique bellifontaine du Cabinet de Clorinde avec le Ballet de Tancrède dans la forêt enchantée où, en toute opposition par rapport au récit du Tasse, Tancrède ne s'effondre pas face au fantôme de Clorinde et brise même l'enchantement de la forêt de Saron. Encore une fois, les protagonistes étaient De Luynes, dans le rôle de Tancrède, et le roi, de nouveau en Godefroy de Bouillon. La nouvelle représentation du corps politique de la Cour était donc bien montrée (Careri 211-214) et passait par l'échec de Clorinde$M a_{i}{ }^{7}$. Reste à rappeler que, cette fois, l'équipe des réalisateurs était privée de son poète principal, Estienne Durand, envoyé à l'échafaud quelques mois auparavant pour crime de lèse-majesté, s'étant mêlé avec deux italiens, les frères Siti, à la publication d'un très violent pamphlet en faveur de la Reinemère et contre le monarque (Laschèvre ; Eustis 191-195 ; Dubost 599-603).

Avec ce Ballet de Tancrède, nous sommes encore une fois en face d'une variation sur l'histoire originelle, qui n'est que la dernière d'une série de réécritures - littéraires, picturales, théâtrales. Dans

Giovanni Careri préfère mettre en relation l'image de Clorinde avec celle de Eleonora Galigai, la femme de Concino Concini qui venait d'être brûlée comme sorcière. Le renversement de l'histoire de Clorinde opéré par le ballet nous semble pourtant plus lisible à la lumière de la tradition tassienne de la cour que nous avons cherché ici à récupérer et il nous semble plus probable que la véritable cible du spectacle était la reine-mère elle-même. 
l'espace d'une vingtaine d'année elles s'étaient emparées du récit du Tasse, permettant à la culture française de le transformer en quelque chose de nouveau. C'était à la fois une façon de le lire et de l'interpréter, mais aussi un code connu par le public, un code qui permettait de faire passer des messages idéologiques. Nous en avons un dernier écho au début des années 1630.

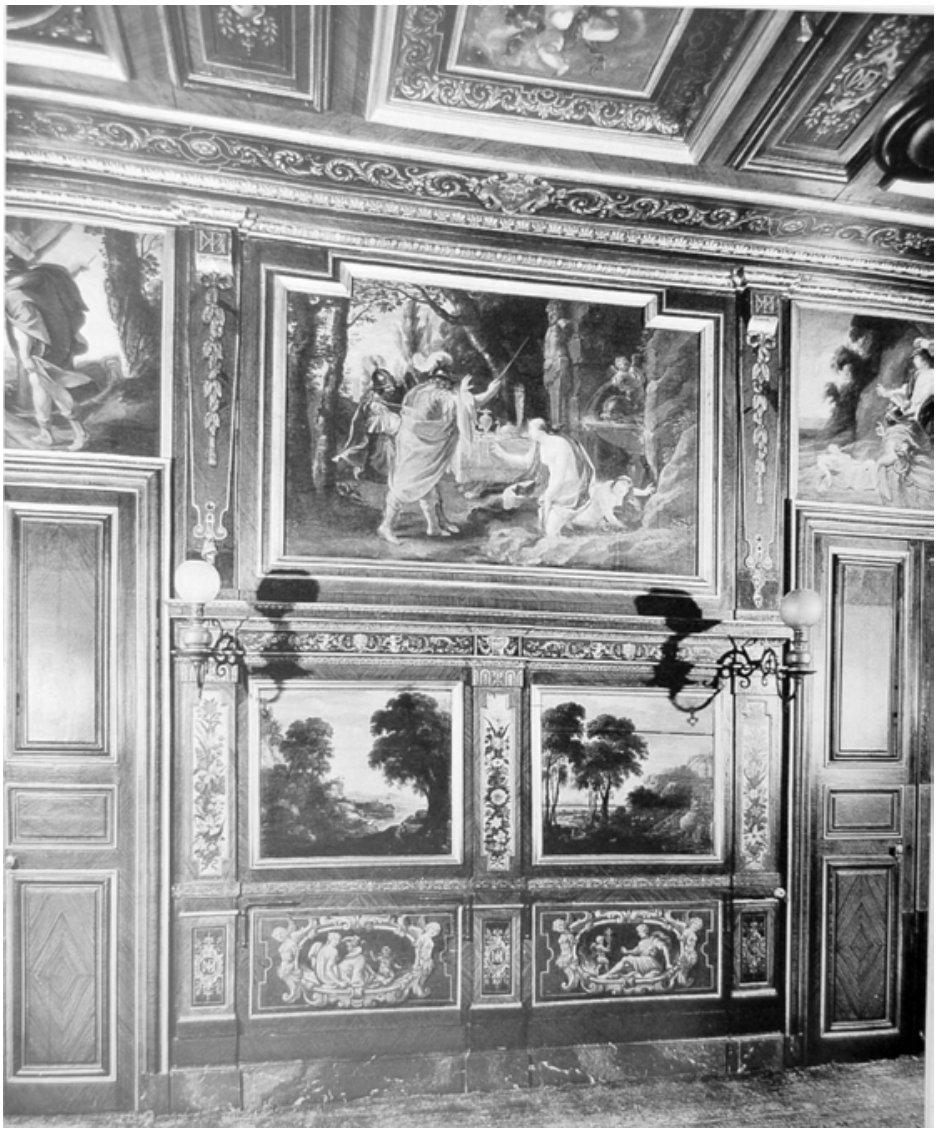

Figure 13 : Simon Vouet, Histoire de Renaud et Armide, 1630-1631, Paris, Collection Guyot de Villeneuve (provenant du Château de Chessy), Musée du Louvre, Documentation.
Entre 1630 et 1631 Simon Vouet peignit une Histoire de Renaud et Armide pour Henri de Fourcy (fig.13), alors surintendant des bâtiments du roi. Or, ce décor avait une destination double : une destination personnelle, dans la galerie du Château de Chessy, la maison de campagne du ministre, et une destination publique dans sa traduction en tapisserie destinée tout d'abord au couple royal et ensuite aux particuliers (Lavalle). En suivant la nouvelle traduction du poème, publiée en 1626 par Jean Baudoin, Simon Vouet revenait à une sorte d' " orthodoxie " tassienne qui se faisait porteuse d'un message positif d'amour, de conversion, de sentiments partagés et de réconciliation dynastique, en exprimant probablement la position d'un ministre proche de Richelieu mais peut-être pas totalement en phase avec l'atmosphère de reddition de 
comptes imposée par le Cardinal au lendemain de la « Journée des Dupes » et de l'éloignement définitif de Marie de Médicis (Quaranta, Deux générations).

Il s'agissait, ici, surtout d'un travail sur les détails. On pense ici à l'insertion, dans l'épisode de l'Enlèvement de Renaud (fig. 14), du grand tournesol au centre du tableau, sorte de véritable « quatrième personnage » dans le chiasme dynamique composé par les trois protagonistes, alors qu'il n'y avait pas de traces d'un tel élément, ni chez le Tasse, ni dans la traduction de Baudoin, où il n'est question que des chaînes de fleurs tissées par Armide pour charmer le chevalier. Mais le tournesol était bien connu de la littérature spirituelle et emblématique, si répandues dans les premières décennies du XVII ${ }^{\mathrm{e}}$ siècle : son aptitude à suivre constamment l'astre du jour évoquait une fidélité assurée, aussi bien dans le domaine religieux que dans le domaine de l'amour nuptial (Choné). Sa présence étalée, bien qu'inattendue dans un tableau qui racontait l'effondrement du héros et le début de son aventure érotique, ne devait pas passer inaperçue, en introduisant un signal qui, troublant le sens affiché de l'épisode, laissait envisager un final positif de l'histoire.

Des tels renvois sont éparpillés partout dans les tableaux du cycle et dans les tapisseries qui en furent tirées : il en est de même, par exemple, pour les couronnes de laurier et les palmiers montrés par des Cupidons dans la scène de la Réconciliation de Renaud et Armide, où elle trouvait finalement sa place. Non seulement, elle offrait une référence tout à fait transparente à la destinée nuptiale du couple, mais elle symbolisait aussi la victoire : une victoire de l'amour sur le vice, du marial sur l'érotique, ainsi que de la réconciliation sur la division, et de la paix sur la guerre. Et ceci,

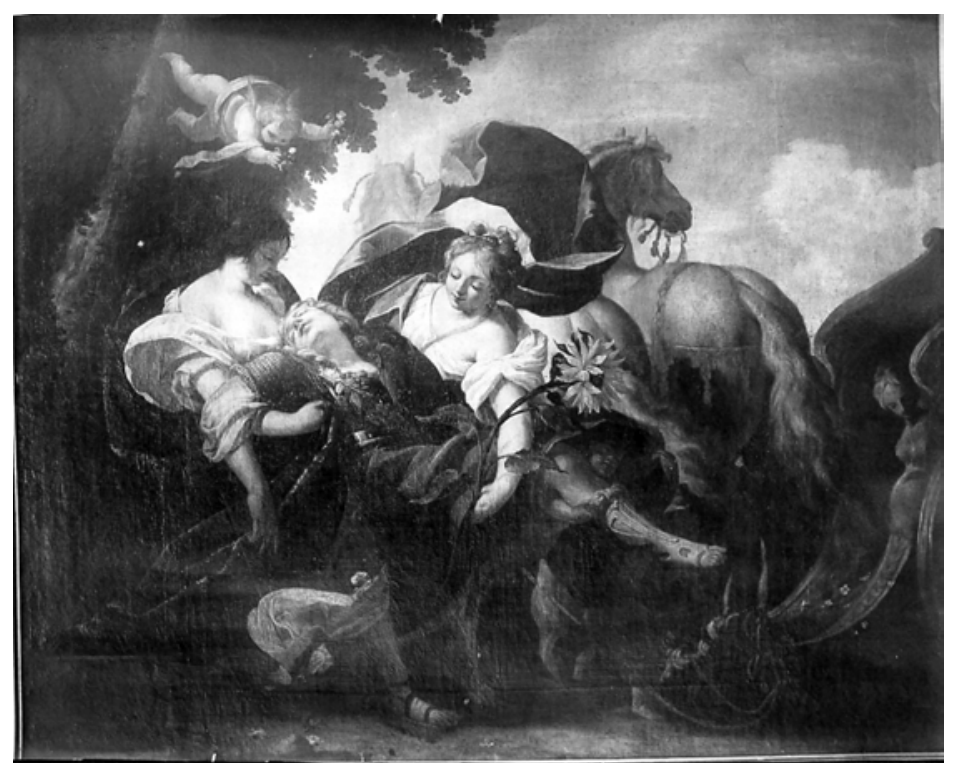

Figure 14 : Simon Vouet, Armide enlève Renaud, 1630-1631, Paris, Collection Guyot de Villeneuve (provenant du Château de Chessy), Musée du Louvre, Documentation. 
tout en se souvenant que le couple royal même s'était trouvé frappé et mis en crise par les événements de 1630 (Quaranta, Deux générations).

Ces insertions opérées par le peintre montrent encore une fois la fonction critique de la mise en image du texte littéraire et son pouvoir de le plier à une lecture idéologique qui en faisait un medium parfait pour un discours à la fois personnel, moral, historique ou politique. Le choix de certains épisodes et l'ajout de certains détails, aussi bien que la faveur donnée au récit original plutôt qu'à l'une de ses adaptations, étaient les outils de la muta eloquentia de l'art pictural. Elle invitait le spectateur à récupérer les différents fragments du discours en faisant des liens entre le tableau qu'il était en train de regarder, l'ensemble du décor, d'autres versions de la même histoire, et aussi des images et des textes appartenant à des traditions différentes.

Mais tout cela n'était possible que grâce à l'existence d'un code commun, connu de tout le monde, bien ancré dans l'imaginaire d'un public qui le reconnaissait comme faisant partie de sa culture spécifique. Le poème du Tasse était devenu l'un de ces codes, une sorte de « langage » composé par des personnages, des épisodes, des situations narratives, dont la modulation pouvait tisser des discours à chaque fois différents. Il l'était devenu grâce à sa vaste et durable fortune auprès du public des lecteurs, qui s'étaient passionnés pour les aventures de ces chevaliers et magiciennes d'espacestemps si reculés et pourtant si proches par leurs troubles et leurs sentiments. Ainsi, alors que le Page Disgracié de François Tristan l'Hermite n'hésitait pas à se battre en duel pour prendre la défense de l'art du Tasse contre un détracteur préférant Virgile (Berregard 133), Monsieur de La Ferté-Sanneterre était, encore au début des années 1640, fier de citer par cœur les stances du poème (Baldensperger 19) et de consacrer à cette histoire le décor majestueux de la grande galerie de son hôtel parisien (Intorno a Poussin). Mais si le chef-d'œuvre italien était devenu une référence connue et partagée, cela n'était arrivé qu'à travers un travail intense d'appropriation, de réécriture, d'adaptation du récit original à des valeurs littéraires, à des codes narratifs, à des attentes du public nouvelles et inédites. En un mot : de la même façon que pour l'image de la reine Marie de Médicis, cela ne s'était produit que suite à une véritable francisation de la Jérusalem Délivrée et de ses protagonistes. 


\section{BiBLIOGRAPHIE}

BALDENSPERGER, Ferdinand. «Le classicisme français et les langues étrangères. " Revue de Littérature Comparée 13 (1933): 14-35.

BALSAMO, Jean. Rencontre de Muses. Italianisme et anti-italianisme dans les lettres françaises à la fin du XVIe siècle. Genève: Librairie Droz, 1992.

BASSANI PACHT, Paola. « Marie de Médicis et ses artistes ». Le « Siècle » de Marie de Médicis, eds. Françoise Graziani, Francesco Solinas. Franco-Italica 21-22 (2002): 81-94.

BÉGUIN Sylvie. « Dessins d'Ambroise Dubois ». L'Eil 135 (mars 1966): 7-15.

BÉGUIN, Sylvie. « Guillaume Dumée. Olindo e Sofronia liberati dal rogo ». Torquato Tasso. Letteratura, musica, teatro, arti figurative, Ed. Andrea Buzzoni, Bologna: Nuova Alfa Editoriale, 1985: n. 71, 238-245.

BERREGARD, Sandrine. Tristan l'Hermite, " héritier » et "précurseur » : imitation et innovation dans la carrier de Tristan l'Hermite. Tubingen: Gunter Narr, 2006.

BERTAUT, Jean. Les Euvres poétiques. Ed. Adolphe Chenevière. Paris: Plon-Nourrit 1890.

BONFAIT, Olivier. Intorno a Poussin. Ideale classico e epopea barocca tra Parigi e Roma. Ed. Olivier Bonfait. Roma : De Luca, 2000.

CARERI, Giovanni. Gestes d'amour et de guerre. La Jérusalem Délivrée, images et affects (XVIe-XVIIe siècle). Paris : Éditions de l'École des Hautes Études en Sciences Sociales, 2005.

CHIAPPELLI, Fredi. Il conoscitore del caos. Una 'vis abdita' nel linguaggio tassesco. Roma: Bulzoni, 1981.

CHONÉ, Paulette. «La prière de l'Hélitrope. Flore au Paradis. Emblématique et vie religieuse aux XVIe et XVIIe siècles. Eds. Paulette Choné, Bénédicte Gaulard. (Glasgow Emblem Studies, 9) Génève: Droz, 2004: 209-230.

CIORANESCU, Alexandre. L'Arioste en France. Des origines à la fin du XVIII' siècle. Paris: Les Éditions Presses Modernes, 1939.

CIORANESCU, Alexandre. Le Masque et le visage: du baroque espagnol au classicisme français. Genève: Librairie Droz, 1983.

CORDELLIER, Dominique. " Dubreuil peintre de « La Franciade » de Ronsard au Château-Neuf de SaintGermain-en-Laye ». Revue du Louvre 35 (1985): 357-378.

COSANDEY, Fanny. La Reine de France, symbole et pouvoir. Paris : Editions Gallimard, 2000

DALLA VALLE, Daniela. Pastorale barocca. Forme e contenuti dal Pastor Fido al dramma pastorale francese. Ravenna: Longo, 1973.

DE MARCO, Maria. «Intorno al testo dell’Excidium Troiae ». Aevum 30 (1, 1956): 36-56.

Discours au vray du ballet dansé par le Roy le dimanche XXIXe jour de janvier M.VI.c XVII. Avec les desseins, tant des machines et apparences differentes, que tous les habits des Masques. Paris: Pierre Ballard, Imprimeur de la Musique du Roy, demeurant ruë Jean de Beauvais, à l'enseigne du mont Parnasse, 1617.

DUBOST, Jean-François. Marie de Médicis. La reine dévoilée. Paris : Biographie Payot, 2009. 
ERLANDE-BRANDENBURG, Alain. «Les appartements de la Reine Mère Marie de Médicis au Louvre », Bulletin de la Société de l'Histoire de l'Art Français, 1965 : 105-113.

EUSTACHE DE THESSALONIQUE. Eustathii archiepiscopi Thessalonicensis Commentarii ad Homeri Iliadem pertinentes ad fidem codicis Laurentiani editi. Volumen primum, Praefationem et commentarios ad libros A-A complectens. Ed. Marchinus van der Valk, Leiden-New York-København-Köln: E.J. Brill, 1971: Rapsoidia B 169/208: 8-13.

EUSTIS, Alvis. Etienne Durand (1585-1618). La Poésie française du premier $17^{e}$ siècle: textes et contextes. 1986. Ed. David Lee Rubin. Charlottesville: Rookwood Press: 2007: 191-195.

Excidium Troiae. Eds. Eimer Bagby Atwood, Virgil Keeble Whitaker. Cambridge (Mass.): The Medieval Academy of America, 1944.

FUMAGALLI, Elena. «Ariosto e Tasso nelle quadrerie medicee del Seicento ». L'Arme e gli amori. La poesia di Ariosto, Tasso e Guarini nell'arte fiorentina del Seicento. 2001. Eds. Elena Fumagalli, Massimiliano Rossi, Riccardo Spinelli. Firenze : Sillabe, 2001: 73-84.

GRAZIANI, Françoise. "Sur le chemin du Tasse. La fidélité du traducteur selon Vigenère, Baudoin et Vion d'Alibray ». L'Arioste et le Tasse en France au XVI siècle (Cahiers V.L. Saulnier, 20). Paris : Éditions Rue d'Ulm, 2003: 203-216.

IANNUZZI, Silvia. « Gli affreschi di Taddeo Zuccari nella Rocca di Bracciano ». Dimore Storiche VII/3 (2002) : 30-39.

JOULET, Pierre, sieur de Chastillon. Les Amours d'Armide, par P. Joulet, Sieur de Chastillon, reveues et augmentées par l'autheur (1596). Paris : Abel L'Angelier, 1608.

KERNSPERN, Sylvain. "Ambroise Dubois: 17. L'Assaut à Jérusalem. 18. Le Baptême de Clorinde ». Marie de Médicis: un gouvernement par les arts. Eds. Paola Bassani Pacht, Thierry Crépin-Leblond, Nicolas Sainte Fare Garnot, Francesco Solinas. Paris-Blois : Somogy Editions d'Art-Château de Blois, 2003: $145-147$.

LACHÈVRE, Frédéric. Estienne Durand, poète ordinaire de Marie de Médicis (1585-1618). Paris : Leclerc, 1905.

LAVALLE, Denis, Simon Vouet et la tapisserie. Vouet. Eds. Jacques Thuillier, Barbara Brejon de Lavergnée, Denis Lavalle. Paris : Réunion des Musées Nationaux, 1990: 512-516.

MACCHIA, Giovanni. La letteratura francese dal Rinascimento al Classicismo (1970). Milano : Rizzoli 2000.

MATTHIEU, Pierre. Histoire de France et des choses mémorables advenues aux provinces estrangères durant sept années de paix du règne de Henry, divisée en sept livres, Paris : J. Métayer, 1605.

McGOWAN, Margaret. L'Art du ballet de cour en France. 1581-1643. Paris : CNRS, 1963.

MILOVANOVICH, Nicolas. «Henri IV à la Bataille d'Arques, le 21 septembre 1589 ». Henri IV à Fontainebleau. Un temps de splendeur. Ed. Vincent Droguet, Paris : Réunion des Musées Nationaux, 2010: 76-79.

NERVÈZE, Antoine de. La Hierusalem Assiegée, où est descrite la délivrance de Sophronie, et d'Olinde, ensemble les amours d'Hermine, de Clorinde et de Tancrède, à l'imitation du s. Torquato Tasso, par A. de Nervèze, seconde édition reveue, corrigée, \& de beaucoup augmentée par l'Autheur. Paris : Anthoine Du Brueil, 1599.

PLAZENET, Laurence. L'Ébahissement et la délectation. Réception comparée et poétiques du roman grec en France et en Angleterre aux XVI et XVII siècles. Paris : Honoré Champion, 1997. 
QUARANTA, Gabriele. «L'Arte del Romanzo. Temi letterari nella pittura francese del Seicento (dal regno di Enrico IV alla reggenza di Anna d'Austria) ». Thèse de doctorat, Cotutelle Sapienza Università di RomaUniversité Paris 1 « Panthéon-Sorbonne», 2013.

. «Deux générations à côté du pouvoir: quelques remarques sur les arts chez les De Fourcy ». Livraisons d'Histoire de l'Architecture 26 (2013) : 105-122.

. «Pentesilea e Clorinda: immagini del non detto da Tasso a Bilivert, a De Nervèze ». Iconologie. Eds. Stefano Pierguidi, Marco Ruffini. Roma : Campisano, à paraître.

ROSENBERG, Pierre, \& Jacques THUILLIER. Laurent de La Hyre (1606-1656): l'homme et l'oeuvre. Genève : Skira, 1988.

SAMOYAULT-VERLET, Colombe. « Ambroise Dubois. L'assalto a Gerusalemme ». Torquato Tasso. Letteratura, musica, teatro, arti figurative, ed. Andrea Buzzoni, Bologna: Nuova Alfa Editoriale, 1985: n. 70, 234-237. . «Ambroise Dubois à Fontainebleau ». Le petit journal des grands expositions 170 (1987).

SARANT, Mylène. «Ambroise Dubois et les Éthiopiques d'Héliodore au XVII ${ }^{\mathrm{e}}$ siècle ». Histoire de l'Art 46 (2000) : 25-37.

SCAILLIEREZ, Cécile. « Le Grand Cabinet de la Reine au Louvre: la part de Gabriel Honnet et de Guillaume Dumée », Revue du Louvre 3 (1989) : 156-163.

SIMPSON, Joyce G. Le Tasse et la littérature et l'art baroques en France, Paris : Librarie A.G. Nizet, 1962.

SOLNON, Jean-François. La Cour de France. Paris : Fayard, 1987.

STEINBERG, Sylvie. "Le mythe des Amazones et son utilisation politique de la Renaissance à la Fronde. Royaume de Fémynie. Pouvoirs, contraintes, espaces de liberté des femmes, de la Renaissance à la Fronde. 1995. Eds. Kathleen Wilson-Chevalier \& Éliane Viennot. Paris : Champion, 1999 : 261-273.

TASSO, Torquato. La Gerusalemme Liberata, 1581. Ed. Bruno Maier, Torino : Biblioteca Universale Rizzoli, 1995.

Il Gonzaga secondo, overo del giuoco, 1581. Dialoghi. Ed. Ezio Raimondi, Firenze : Sansoni, 1958: vol. II, t. 1.

VÉRON-DENISE, Danièle. «Le cabinet de Théagène et Chariclée ». Henri IV à Fontainebleau. Un temps de splendeur. Ed. Vincent Droguet, Paris : Réunion des Musées Nationaux, 2010: 94-111.

VIENNOT, Eliane. « Des 'Femmes d'Etat' au XVI e siècle: les princesses de la Ligue et l'écriture de l'histoire ». Femmes et pouvoirs sous l'Ancien Régime. Eds. Danielle Haase-Dubosc, Eliane Vierdot. Paris-Marseille : Payot-Rivages, 1991: 439-457.

La France, les femmes et le pouvoir. L'invention de la loi salique, $V^{E}-X V T^{e}$ siècle. Paris : Perrin, 2006.

WIRTH, Stanislas. «La galerie de la Reine, dite de Diane ». Henri IV à Fontainebleau. Un temps de splendeur. Ed. Vincent Droguet. Paris : Réunion des Musées Nationaux, 2010 : 63-69.

«Le cabinet de Tacrède et Clorinde ». Henri IV à Fontainebleau. Un temps de splendeur. Ed. Vincent Droguet. Paris : Réunion des Musées Nationaux, 2010 : 82-93.

ZATTI, Sergio. L'ombra del Tasso. Epica e romanzo nel Cinquecento. Milano : Bruno Mondadori, 1996. 


\section{ILLUSTRATIONS}

Figure 1: Fontainebleau, Cabinet de Clorinde, Restitution de la disposition originaire des tableaux, par C. Samoyault-Verlet $(1987,1)$.

Figure 2: Ambroise Dubois, Tancrède et Clorinde à la fontaine, 1604-1606, huile sur toile, Château de Fontainebleau, F.1980-3.

Figure 3: Antoine de Nervèze, La Hierusalem Assiegée (...), Paris, chez Anthoine Du Brueil, 1599, page de frontispice (Paris, Arsenal, 8-BL- 18456).

Figure 4: Ambroise Dubois, Assaut à Jérusalem, 1604-1605, huile sur toile, Château de Fontainebleau, F.3232.C.

Figure 5: Entourage de François Bunel, Henri IV à la bataille d'Arques, XVII ${ }^{\mathrm{e}}$ siècle, huile sur bois, Pau, Musée National du Château, P.2007.6.1.

Figure 6: Ambroise Dubois, Clorinde devant Aladin, 1604-1606, huile sur toile, Château de Fontainebleau, INV 4159.

Figure 7 : Ambroise Dubois, Baptême de Clorinde, 1604-1606, huile sur toile, Château de Fontainebleau, F.1980-4.

Figure 8: Anonyme, Lamentation de Tancrède sur le corps de Clorinde, Figure dans Antoine de Nervèze, La Hierusalem Assiegée, Paris, chez Anthoine Du Brueil 1599, c. 127r (Paris, Arsenal, 8-BL-18456).

Figure 9: Anonyme, Tancrède pleurant sur la tombe de Clorinde, Figure dans Antoine de Nervèze, La Hierusalem Assiegée, Paris, chez Anthoine Du Brueil, 1599, c. 132v (Paris, Arsenal, 8-BL-18456).

Figure 10: Laurent de La Hyre, Tancrède pleurant sur la tombe de Clorinde, Ann Arbor, The University of Michigan Museum of Art, 1970/2-89.

Figure 11 : Laurent de La Hyre, Tancrède dans la forêt enchantée (?), ici proposé comme Tancrède et Clorinde à la fontaine, Ann Arbor, The University of Michigan Museum of Art, 1970/2-88.

Figure 12 : Gabriel Honnet, Sophronie qui s'accuse devant Aladin, vers 1613- 1615, dessin, Paris, ENSBA, M.904.

Figure 13: Simon Vouet, Histoire de Renaud et Armide, 1630-1631, Paris, Collection Guyot de Villeneuve (provenant du Château de Chessy), Musée du Louvre, Documentation.

Figure 14 : Simon Vouet, Armide enlève Renaud, 1630-1631, Paris, Collection Guyot de Villeneuve (provenant du Château de Chessy), Musée du Louvre, Documentation. 